\title{
NOMENCLATURE PROPOSÉE POUR LA CHÉTOTAXIE DES CERCAIRES DE PLAgIORCHIATA
}

\author{
Ch. BAYSSADE-DUFOUR*, J.-L. ALBARET*, \\ B. GRABDA-KAZUBSKA**, A. G. GHABAUD*
}

RÉSUMÉ. Le schéma du système nerveux d'une cercaire de Plagiorchiata est réalisé en synthétisant les données bibliographiques disponibles.

En superposant à ce schéma la disposition des sensilles d'une cercaire déterminée (Omphalometra flexuosa), l'innervation de chaque sensille ou groupe de sensilles peut être précisée. Cela facilite la compréhension des phénomènes qui dirigent l'évolution de la chétotaxie, ce qui est essentiel pour une étude cladistique du groupe.

Quelques modifications sont proposées dans la nomenclature des sensilles.

Mots-clés : Plagiorchiata. Sensilles des cercaires. Nomenclature.

\section{Proposed nomenclature for Plagiorchiata cercariae chaetotaxy.}

SUMMARY. A diagram of the nervous system of Plagiorchiata cercaria has been drawn according to the relevant bibliographical data.

By superposing on this diagram the drawing of sensillae in a given cercaria (Omphalometre flexuosa) the innervation of each sensilla or group of sensillae may be determined. This facilitates the understanding of the evolutionary trends of chaetotaxy, which is essential for a cladistic study of the group. Some modifications of the nomenclature of sensillae are proposed.

Key-words : Plagiorchiata. Cercarial papillae. Nomenclature.

\section{Introduction}

De nombreux travaux ont montré l'intérêt de la systématique fondée sur la chétotaxie des cercaires, aussi bien pour les problèmes de phylogénie, à l'échelle générique et supragénérique que pour les problèmes de spéciation, à l'échelle spécifique ou infraspécifique.

La nomenclature des soies proposée par Richard (1968 et 1971) repose sur l'hypothèse de sensilles situées à l'intersection de six troncs nerveux longitudinaux

* Laboratoire de Zoologie (Vers), associé au CNRS, Muséum national d'Histoire naturelle, 61 , rue de Buffon, F 75231 Paris Cedex 05.

** W. Stefanski, Institute of the Parasitology of the Polish Academy of Sciences, L. Pasteur 3, P. O. Box 153, 00-973 Warszawa, Poland.

Accepté le 26 avril 1989. 
(2 ventraux, 2 latéraux et 2 dorsaux) et de onze commissures transversales (4 céphaliques et 7 corporelles).

Ce système élémentaire a permis de faire face à la plupart des structures rencontrées, depuis les plus simples (Schistosomes) jusqu'aux plus complexes (Paramphistomes).

Cependant les précisions nécessaires pour les études cladistiques font qu'il est souhaitable de comprendre quelle est l'évolution de chaque soie ou groupe de soies. Il faut donc préciser quelles sont les particularités de chaque superfamille pour tenter d'élucider l'innervation exacte des papilles.

L'objet de ce travail est donc, dans le cas des Plagiorchiata, d'établir, à partir des données bibliographiques, quelle est l'anatomie du système nerveux, quelle est l'innervation des papilles et d'en déduire une nomenclature de celles-ci qui s'écarte aussi peu que possible de celle qui a été précédemment établie.

Système nerveux D'une cercaire de Plagiorchiata

Les travaux utilisés sont ceux de Gaffron (1884) sur juvénile d'Orchipedidae (Dicrocoelioidea), de Bettendorf (1897) sur métacercaire de Brachylaimidae, de Zailer (1914) sur adulte de Plagiorchiidae, de Niewiadomska et Moczon (1982) sur cercaire de Diplostomatidae (Strigeoidea), de Grabda-Kazubska et Moczon (1981) sur cercaire de Plagiorchiidae et (1988) sur cercaire d'Echinostomatidae. Le système nerveux paraît remarquablement stable quelle que soit l'espèce et quel que soit le stade étudié ; la synthèse des documents disponibles permet de réaliser les figures 1 à 3.

On peut y noter :

a - la région céphalique comporte deux commissures :

- un large anneau antérieur sur lequel se trouvent les papilles des cercles CI, CII et la partie interne de CIII, selon la nomenclature de Richard (1971), ce qui correspond au phénomène évolutif normal de condensation des papilles autour de la bouche,

-- un mince anneau sur lequel se trouvent les papilles de la partie externe de CIII que nous appelons papilles du cycle CIV dans la nomenclature proposée,

b - la région corporelle porte six commissures ;

c - l'acétabulum est innervé par des rameaux issus des deux troncs ventraux et peut être des deux commissures AIII et PI qui l'entourent. tabulum.

Il n'y a pas de commissure M entre AIII et PI pour l'innervation de l'acé-

d - la queue est innervée par un filet naissant sur la dernière commissure corporelle ventrale ;

e - la partie antérieure, pré-ganglionnaire, de chacun des troncs nerveux longitudinaux est liée à la partie postérieure post-ganglionnaire correspondante par un filet connectif. Nous admettons donc l'existence de six connectifs surajoutés aux troncs longitudinaux. 

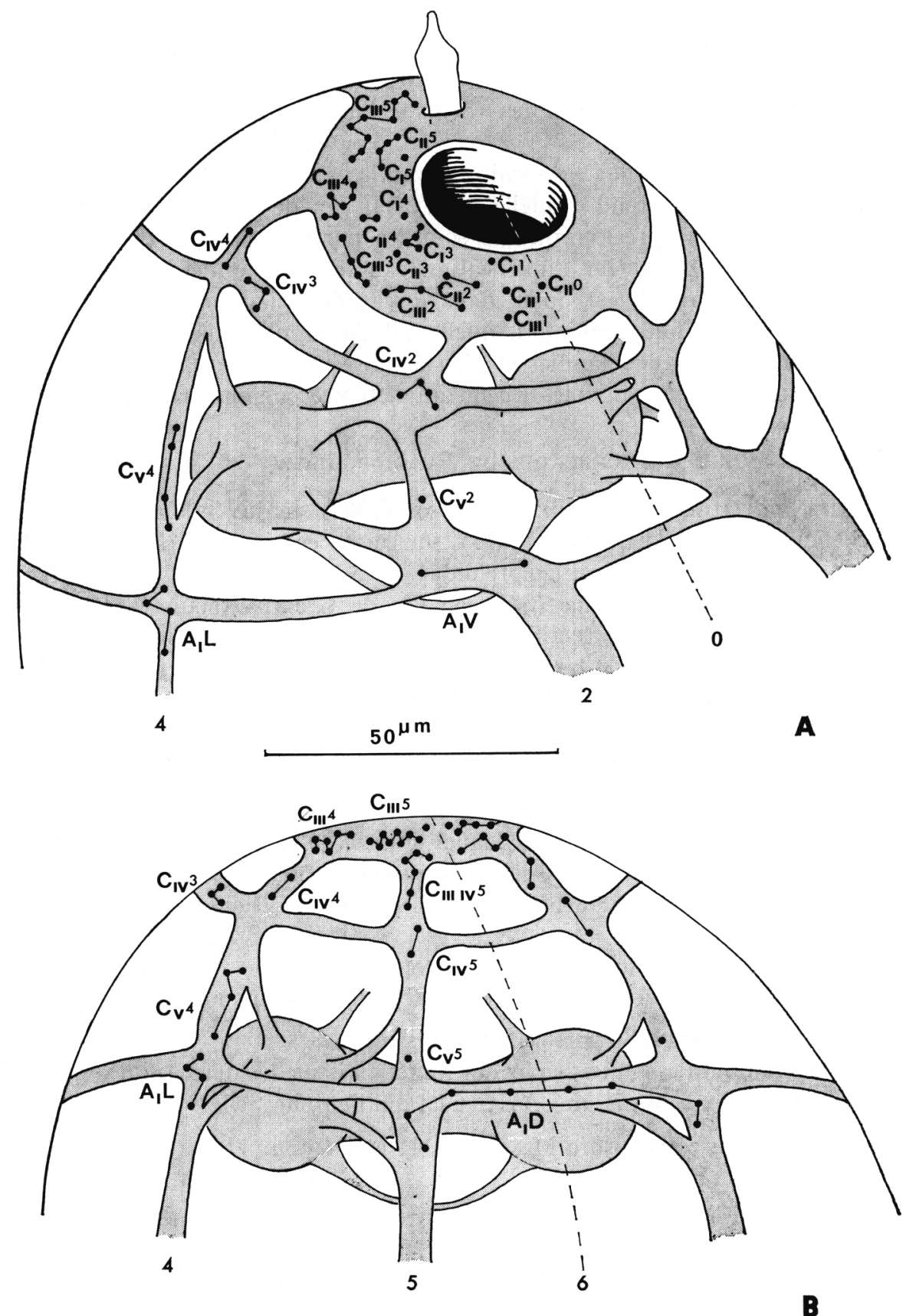

FIG. 1. - Cercaire de Omphalometra flexuosa. Disposition et innervation présumée des papilles céphaliques et des papilles du cycle A I. A : Vue ventro-latérale. B : Vue dorso-latérale. 


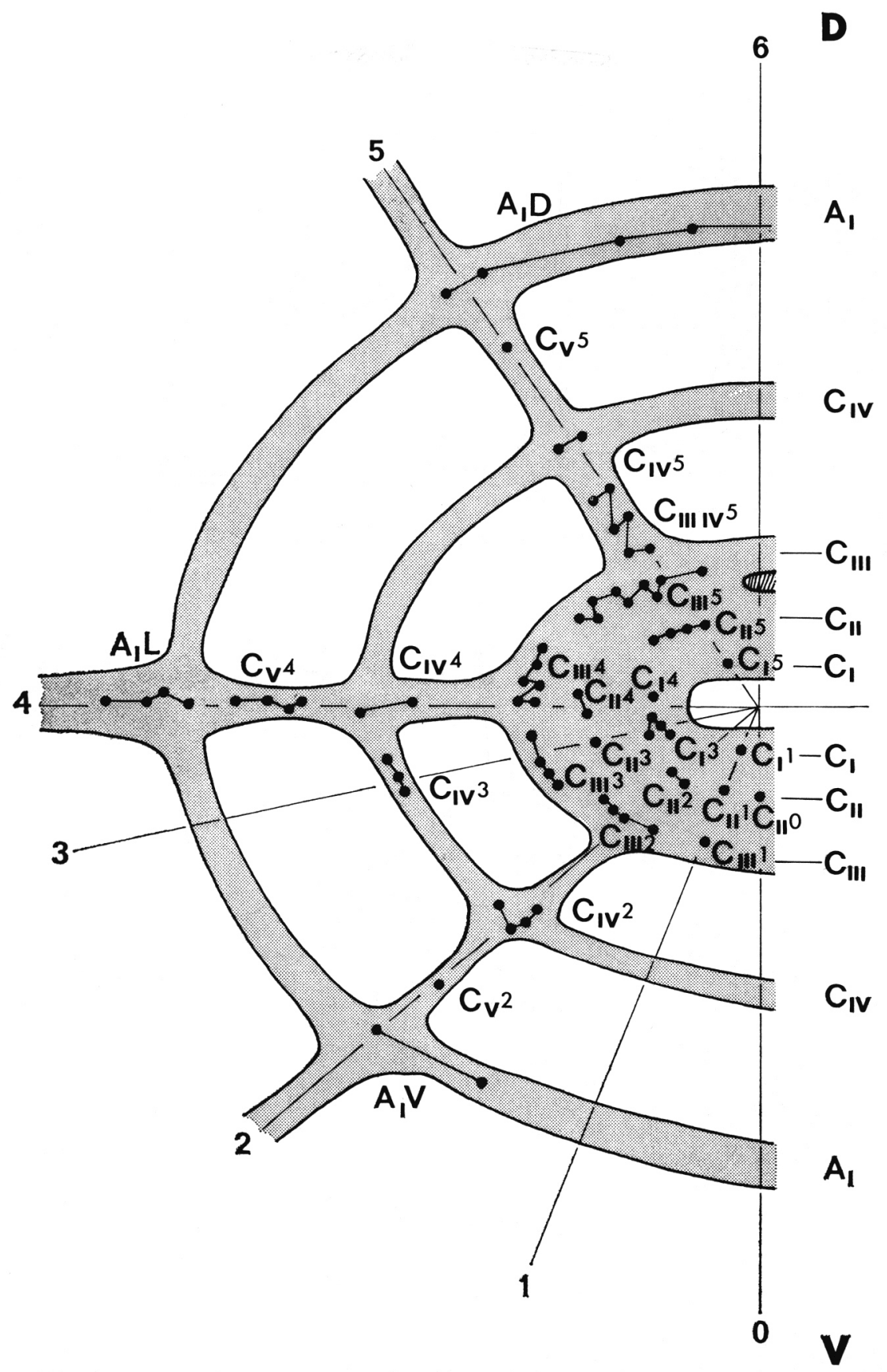

FIg. 2. - Cercaire de Omphalometra flexuosa. Diagramme, disposition et innervation présumée des papilles céphaliques et des papilles du cycle AI. Vue apicale. D : face dorsale V : face ventrale. 


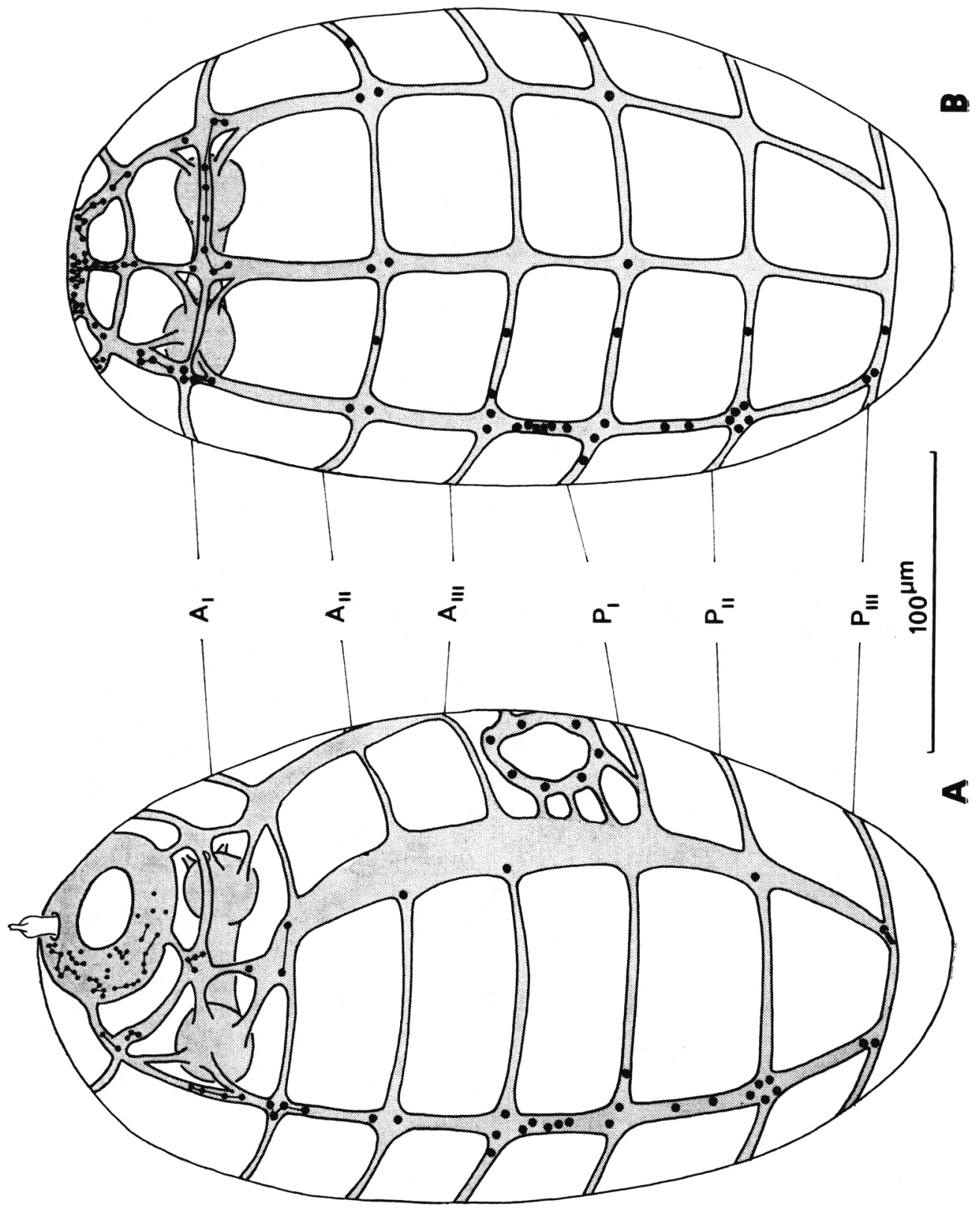

FIg. 3. - Cercaire de Omphalometra flexuosa. Disposition et innervation présumée d'une partiedes papilles céphaliques et des papilles corporelles. A : Vue ventro-latérale. B : Vue dorsolatérale. 


\section{INNERVATION DES PAPILLES}

Les figures 1 et 2 présentent en superposition les sensilles et les nerfs de la région céphalique d'une cercaire de Omphalometra flexuosa Odening, 1960 (= Cercaria sp. 6 Richard, 1971) prise comme exemple de Plagiorchiata.

Il apparaît que la plupart des sensilles sont concentrées sur certains axes longitudinaux correspondant aux nerfs sous-jacents dirigés vers la bouche. Cela entraîne une répartition radiale péribuccale qui fait que l'on peut, de chaque côté, distinguer cinq secteurs : 1 et 2 ventraux, 3 et 4 latéraux et 5 dorsal.

Chaque papille ou groupe de papilles céphaliques (C) est donc caractérisé par sa commissure (I, II, III ou IV), et par son secteur (1, 2, 3, 4 ou 5), les chiffres 0 et 6 étant employés pour désigner les papilles médio-inférieures et médio-supérieures, lorsque elles existent.

TABleau I. - Tableau des correspondances entre les différentes nomenclatures proposées.

\begin{tabular}{|c|c|c|c|c|c|}
\hline \begin{tabular}{|l|}
$\begin{array}{l}\text { Richard } \\
1971\end{array}$ \\
\end{tabular} & \begin{tabular}{|l} 
Bayssade-Dufour \\
1979 \\
\end{tabular} & $\begin{array}{l}\text { Nomenclature } \\
\text { proposée }\end{array}$ & \begin{tabular}{|l|} 
Troncs \\
nerveux
\end{tabular} & Secteurs & $\begin{array}{l}\text { Nombre de } \\
\text { papilles }\end{array}$ \\
\hline CIV & CIY & CI1 & TY & 1 & $\pi$ \\
\hline $\mathrm{CIL}$ & CIL & ci3 & L & 3 & 4 \\
\hline C $10 \ldots d 2$ & $C 10$ & C 14 & L & 4 & 1 \\
\hline$C / D \ldots d 1$ & $C 10$ & C15 & D & 5 & 1 \\
\hline CIIO & C110 & cilo 0 & V & 0 & $1 / 2$ \\
\hline CIII & $c \| 11$ & C II 1 & v & 1 & 1 \\
\hline CII2 & c112 & C 112 & V & 2 & 2 \\
\hline $\mathrm{C} 113$ & $c 113$ & C 113 & L & 3 & 1 \\
\hline CII 4 & CII 4 & CII 4 & L & 4 & 2 \\
\hline$S t 1($ ant $)+S t$ V & $\mathrm{H} 2$ & CII 5 & D & 5 & 4 \\
\hline CIII 1 & CIIII & CIIII & V & 1 & 1 \\
\hline C III 2(int) & CIII 2 & CIII 2 & V & 2 & 4 \\
\hline CIII 3(int) & CIII 3(part) & CIII 3 & L & 3 & 4 \\
\hline$s+2($ int $)$ & $\mathrm{HL} 3$ & CIII 4 & L & 4 & 6 \\
\hline$S t 1$ (post)+St D & H3 & CIII 5 & D & 5 & 9* \\
\hline C III 2(ext) & CIII2 & CIV 2 & V & 2 & 4 \\
\hline C III 3(ext) & cIII 3 & c IV 3 & L & 3 & 3 \\
\hline St 2(ext) & HL 3 (part) & C IV 4 & $\mathrm{~L}$ & 4 & 2 \\
\hline St D L(ant) & H4 & CIII-IY 5 & D & 5 & $2+2+2$ \\
\hline St DL( post) & H4 & C IY 5 & D & 5 & 2 \\
\hline$A \mid V(a n t)$ & AIV & C V 2 & Conn.Y & 2 & 1 \\
\hline$A \mid L$ (ant) & $|A| L$ & CV 4 & Conn.L & 4 & 4 \\
\hline$A \mid D$ (ant) & $A 1 D$ & CV 5 & Conn.D & 5 & 1 \\
\hline
\end{tabular}

* entre C III 5 et C III-IY 5, huit éléments sombres semblent correspondre à des orifices glandulaires 
Les groupes de papilles désignés par Richard (1971) et par Bayssade-Dufour (1979) sous les noms de CIII2 et CIII3 occupent un territoire qui se superpose à deux anneaux commissuraux différents, un gros, interne, pour les soies antérieures, et un mince, externe, pour les soies postérieures, nous préférons donc scinder ces groupes en une portion III et une portion IV.

De la même façon, les soies nommées précédemment "soies du stylet "sont désignées ici, selon le territoire qu'elles occupent, en quatre groupes au lieu de trois.

En arrière des deux anneaux correspondant respectivement aux commissures $(I+$ II + III) et (IV) se trouvent plusieurs groupes de papilles qui paraissent se superposer aux nerfs connectifs. Pour simplifier l'écriture et ne pas créer une nouvelle rubrique ces sensilles seront désignées comme " $\mathrm{CV}$ " bien que dans notre interprétation ils correspondent à une innervation par les nerfs connectifs et non par une commissure céphalique.

Pour la région somatique, post-ganglionnaire, aucune modification ne paraît nécessaire, à l'exception de la commissure M qui doit être supprimée (fig. 3).

La correspondance entre les nomenclatures précédentes et celle qui est proposée est indiquée dans le tableau $I$.

\section{RÉRÉRENCES}

Bayssade-Dufour Ch. : L'appareil sensoriel des cercaires et la systématique des Trématodes Digénétiques. Mém. Mus. natn. Hist. nat., sér. A. Zool., 1979, 113, 1-81.

Bettendorf H. : Ueber musculatur und Sinneszellen der Trematoden. Zool. Jahrb. Yena Anat., 1897, 10, 308-358.

Gaffron E. : Zum Nervensystem der Trematoden. Zool. Beitr. heraus geg. v. A. Schneider, 1884, $1,109-115$.

Grabda-Kazubska B., Moczon T. : Nervous system and chaetotaxy in the Cercaria of Haplometra cylindracea (Zeder, 1800) (Digenea, Plagiorchiidae). Z. Parasitenk, 1981, 6.5, 53-61.

Grabda-Kazubska B., Moczon T. : The nervous system of Echinoparyphium recurvatum (Linstow, 1873) cercaria and adult (Digenea, Echinostomatidae). Acta parasit. pol., 1988, 33, 177-184.

Niewiadomska K., Moczon T. : The nervous system of Diplostomum pseudospathaceum Niewiadomska (Digenea, Diplostomatidae). Z. Parasitenk, 1982, 68, 295-304.

Richard J. : La chétotaxie des cercaires. Valeur systématique. C. R. Acad. Sc. Paris, 1968, $266,371-374$.

Richard J. : La chétotaxie des cercaires. Valeur systématique et phylétique. Mém. Mus. natn. Hist. nat., sér. A, 1971, Zool., 6\%, 1-179.

ZAILER O. : Zur kenntniss der Anatomie der Muskulatur und des Nervensystems der Trematoden. Zool. Anzeiger, 1914, 44, 385-396. 